\title{
Force-velocity relationship profile of elbow flexors in male gymnasts
}

\author{
Miyuki Nakatani $^{1,2}$, ${\text { Kensuke } \text { Murata }^{2} \text {, Hiroaki Kanehisa }}^{3}$, Yohei Takai ${ }^{\text {Corresp. } 2}$ \\ ${ }^{1}$ The Center for Liberal Arts, Meiji Gakuin University, Yokohama, Kanagawa, Japan \\ 2 National Institute of Fitness and Sports in Kanoya, Kanoya, Kagosima, Japan \\ 3 Faculty of Sport and Health Science, Ritsumeikan University, Kusatsu, Shiga, Japan \\ Corresponding Author: Yohei Takai \\ Email address: y-takai@nifs-k.ac.jp
}

Background. The theoretical maximum force $\left(\mathrm{F}_{0}\right)$, velocity $\left(\mathrm{V}_{0}\right)$, and power $\left(\mathrm{P}_{\max }\right)$ of athletes calculated from the relationship between force and velocity (F-V relationship) and the slope of the F-V relationship, reflect their competitive and training activity profiles. Evaluating the F-V relationship of athletes facilitates categorizing the profiles of dynamic muscle functions in relation to long-term sport-specific training. For gymnastics, however, no studies have tried to examine the profiles of F-V relation and power output for upper limb muscles in relation to the muscularity, while the use of the upper extremities in this sport is very unique as described earlier.

Purpose. It was hypothesized that the F-V relationship of the elbow flexion in gymnasts might be characterized by low capacity for generating explosive force, notably in terms of the force normalized to muscle size.

Methods. The $F_{0}, V_{0}$, and $P_{\max }$ derived from the force-velocity relationship during explosive elbow flexion against six different loads (unloaded condition, $15,30,45,60$, and $75 \%$ of maximal voluntary isometric elbow flexion force $\left(\mathrm{MVF}_{\mathrm{EF}}\right)$ ) for 16 gymnasts $(\mathrm{GYM})$ and 22 judo athletes (JD). $\mathrm{F}_{0}$ and $\mathrm{P}_{\max }$ were expressed as values relative to the cross-sectional area index $\left(\mathrm{CSA}_{\text {index }}\right)$ of elbow flexors $\left(\mathrm{F}_{0} / \mathrm{CSA}_{\text {index }}\right.$ and $\mathrm{P}_{\text {max }} / \mathrm{CSA}_{\text {index }}$ respectively), which was calculated from muscle thickness in the anterior upper arm. The electromyogram (EMG) activities of the biceps brachii (BB) during the maximal isometric and dynamic tasks were also determined.

Results. There were no significant differences in $\mathrm{CSA}_{\text {index }}$ of elbow flexors between GYM and JD. $\mathrm{MVF}_{\mathrm{EE}} / \mathrm{CSA}_{\text {index }}$ for GYM was significantly lower than that for JD. Force was linearly associated with velocity in the dynamic elbow flexion for all the participants ( $r=-0.997$ to -0.905 for GYM, $r=-0.998$ to -0.840 for JD). $F_{0}, F_{0} / C S S_{\text {index }}, V_{0}, P_{\text {max }}, P_{\text {max }} / C S S A_{\text {index }}$, and $M \mathrm{MF}_{E F}$ were significantly lower in GYM than in JD. The activity levels of BB during the dynamic tasks tended to be lower in GYM than in JD at load of $<45 \% \mathrm{MVC}$.

Conclusion. Gymnasts cannot generate explosive elbow flexion force corresponding to their muscle size. This may be due to low neuromuscular activities during the maximal dynamic tasks against relatively low loads. 


\section{Title:}

2 Force-velocity relationship profile of elbow flexors in male gymnasts

4 Short title:

5 Force-velocity profile in male gymnasts

\section{Authors:}

8 Miyuki Nakatani ${ }^{1,2)}$, Kensuke Murata ${ }^{2)}$, Hiroaki Kanehisa ${ }^{3)}$, Yohei Takai ${ }^{2)}$

10 1) The Center for Liberal Arts, Meiji Gakuin University, 1518 Kamikurata, Totsuka-ku, Yokohama, 11 Kanagawa, 244-8539, Japan.

12 2) National Institute of Fitness and Sports in Kanoya, 1 Shiromizu, Kanoya, Kagoshima, 891-2393, 13 Japan

14 3) Faculty of Sport and Health Science, Ritsumeikan University, 1-1-1 Noji-higashi, Kusatsu, 15 Shiga, 525-8577, Japan

17 Mailing address of the corresponding author:

18 Yohei Takai

19 National Institute of Fitness and Sports in Kanoya

201 Shiromizu, Kanoya, Kagoshima, 891- 2393 Japan

21 Tel: +81994-46-4992

22 Fax: +81994-46-4992

23 e-mail: y-takai@nifs-k.ac.jp 


\section{Abstract}

26 Background. The theoretical maximum force $\left(\mathrm{F}_{0}\right)$, velocity $\left(\mathrm{V}_{0}\right)$, and power $\left(\mathrm{P}_{\max }\right)$ of athletes calculated from the relationship between force and velocity ( $\mathrm{F}-\mathrm{V}$ relationship) and the slope of the F-V relationship, reflect their competitive and training activity profiles. Evaluating the F-V relationship of athletes facilitates categorizing the profiles of dynamic muscle functions in relation to long-term sport-specific training. For gymnastics, however, no studies have tried to examine the profiles of F-V relation and power output for upper limb muscles in relation to the muscularity, while the use of the upper extremities in this sport is very unique as described earlier.

Purpose. It was hypothesized that the F-V relationship of the elbow flexion in gymnasts might be characterized by low capacity for generating explosive force, notably in terms of the force normalized to muscle size.

Methods. The $\mathrm{F}_{0}, \mathrm{~V}_{0}$, and $\mathrm{P}_{\max }$ derived from the force-velocity relationship during explosive elbow flexion against six different loads (unloaded condition, 15, 30, 45, 60, and 75\% of maximal voluntary isometric elbow flexion force $\left.\left(\mathrm{MVF}_{\mathrm{EF}}\right)\right)$ for 16 gymnasts $(\mathrm{GYM})$ and 22 judo athletes (JD). $\mathrm{F}_{0}$ and $\mathrm{P}_{\max }$ were expressed as values relative to the cross-sectional area index $\left(\mathrm{CSA}_{\text {index }}\right)$ of elbow flexors $\left(\mathrm{F}_{0} / \mathrm{CSA}_{\text {index }}\right.$ and $\mathrm{P}_{\max } / \mathrm{CSA}_{\text {index }}$, respectively), which was calculated from muscle thickness in the anterior upper arm. The electromyogram (EMG) activities of the biceps brachii (BB) during the maximal isometric and dynamic tasks were also determined.

Results. There were no significant differences in $\mathrm{CSA}_{\text {index }}$ of elbow flexors between GYM and JD. $\mathrm{MVF}_{\mathrm{EF}} / \mathrm{CSA}_{\text {index }}$ for GYM was significantly lower than that for JD. Force was linearly associated with velocity in the dynamic elbow flexion for all the participants ( $r=-0.997$ to -0.905 for GYM, $r=-0.998$ to -0.840 for JD). $\mathrm{F}_{0}, \mathrm{~F}_{0} / \mathrm{CSA}_{\text {index }}, \mathrm{V}_{0}, \mathrm{P}_{\max }, \mathrm{P}_{\max } / \mathrm{CSA}_{\text {index }}$, and $\mathrm{MVF}_{\mathrm{EF}}$ were significantly lower in GYM than in JD. The activity levels of BB during the dynamic tasks tended to be lower in GYM than in JD at load of $<45 \%$ MVC.

Conclusion. Gymnasts cannot generate explosive elbow flexion force corresponding to their muscle size. This may be due to low neuromuscular activities during the maximal dynamic tasks against relatively low loads. 


\section{Introduction}

The competitive events of artistic gymnastics for men consist of "floor," "rings," "pommel horse," "long horse," "parallel bars," and the "horizontal bar." Gymnastic training involves, on average, 102 impacts per session, and loads of 1.5 to 3.6 times the bodyweight on the upper extremity when performing the actions such as hurdle step, round-off, back handspring, forward handspring, and pommel of young gymnasts (Daly et al. 1999). During the handstand and the swallow on the rings, the electromyogram amplitude of the biceps brachii, normalized to that during maximal voluntary contraction (MVC) is as high as 50-80\% (Bernasconi et al. 2009; Kochanowicz et al. 2018b). Gymnasts are frequently required to support their body mass and control body balance by using the upper extremities while overcoming repetitive high-impact loadings (DiFiori et al. 2002). In other words, gymnasts repeat highly intense and sustained upper arm muscle activities during competitions and training. The unique use of upper limb muscles by gymnasts is one factor yielding the hypertrophied muscularity of this segment (Claessens et al. 1991; Ichinose et al. 1998; Spenst et al. 1993; Takai et al. 2018)

The muscle size (e.g. muscle cross-sectional area and muscle volume) is a significant determinant of force- and power-generating capacities of the upper arms (Fukunaga et al. 2001; Wakahara et al. 2013). There is little information from earlier studies on the isometric and dynamic strength of the upper limb muscles of gymnasts. Only three studies have provided data on isometric and dynamic strength of gymnasts (Kochanowicz et al. 2018b; Kochanowicz et al. 2019; Niespodzinski et al. 2018), but their findings are mutually contradictory. One study has found higher isometric elbow flexor strength in male gymnasts compared to untrained people (Niespodzinski et al. 2018), but other studies have reported the opposite result (Kochanowicz et al. 2018b; Kochanowicz et al. 2019). The earlier studies have attempted to clarify force-generating capacity of gymnasts compared to individuals who have not experienced regular sport-specific training. In general, well-trained individuals have greater muscle size as well as voluntary strength compared to sedentary individuals (Alway et al. 1990; Sale et al. 1987). For clarifying the profiles of force- and power-generating capacities in gymnasts, therefore, it is necessary to compare them with well-trained individuals with similar upper limb muscularity as that of gymnasts.

Many studies aiming to evaluate the dynamic muscle function of athletes have determined the force-velocity $(\mathrm{F}-\mathrm{V})$ and/or the load-power relationship of explosive multi-joint movements such as the bench press, throwing, jumping, and cycling, which is obtained by using loads relative to one repetition maximum (1RM) of the task or body mass (Asci \& Acikada 2007; Baker 2001; Baker \& Newton 2006; Bozic \& Bacvarevic 2018; Giroux et al. 2016; Izquierdo et al. 2002; McBride et al. 1999; Vuk et al. 2012). Their findings suggest that the theoretical maximum force $\left(\mathrm{F}_{0}\right)$, velocity $\left(\mathrm{V}_{0}\right)$, and power $\left(\mathrm{P}_{\max }\right)$ of athletes calculated from the $\mathrm{F}-\mathrm{V}$ relationship and the slope of the F-V relationship, reflect their competitive and training activity profiles (Bozic \& Bacvarevic 
89 2018; Giroux et al. 2016; Izquierdo et al. 2002; McBride et al. 1999). For example, Bozic \& 90 Bacvarevic (2018) found that in maximal sprints on a leg cycle ergometer, wrestlers and judo 91 athletes showed higher $\mathrm{F}_{0}$ with force-oriented slope, which means steeper slope, and the sprinters 92 higher $\mathrm{V}_{0}$. Evaluating the $\mathrm{F}-\mathrm{V}$ relationship of athletes facilitates categorizing the profiles of 93 dynamic muscle functions in relation to long-term sport-specific training. For gymnastics, 94 however, no studies have tried to examine the profiles of $\mathrm{F}-\mathrm{V}$ relation and power output for upper 95 limb muscles in relation to the muscularity, while the use of the upper extremities in this sport is very unique as described earlier.

Ballistic and/or explosive exercises are highly useful for improving power production (Cormie et al. 2011). However, such training-induced changes in maximal power production and F-V relationships vary with the magnitude of the adapted load and the actual movement velocity during exercise (Cormie et al. 2011; Djuric et al. 2016; Jimenez-Reyes et al. 2016; Kaneko et al. 1984; McBride et al. 1999). As described above, competitive and training activities for gymnasts can be characterized as highly intense and sustained muscle contractions to support the body mass and the successful control of body balance. A training modality with intense and sustained muscle contractions (lasting $3 \mathrm{~s}$ at $75 \%$ of $\mathrm{MVC}$ ) is less effective for explosive muscle functions and activation compared to explosive contractions at $>80 \%$ of MVC lasting $<1 \mathrm{~s} \mathrm{(Balshaw} \mathrm{et} \mathrm{al.} \mathrm{2016).}$ No significant difference in isometric MVC torque of elbow flexion has been reported between gymnasts and untrained individuals, in spite of greater arm lean tissue mass in gymnasts (Kochanowicz et al. 2018b). Based on these findings, we can hypothesize that as a result of longterm sport-specific training, the F-V relationship of the upper limb muscles in gymnasts might be characterized by low capacity for generating explosive force, notably in terms of the force normalized to muscle size, i.e., muscle quality. This study aimed to clarify the profile of the F-V relationship of elbow flexors in male gymnasts. 


\section{Methods}

\section{Participants}

116 Thirty-eight adult men voluntarily participated in this study. The means and standard deviations 117 (SDs) for age, body height, and body mass were $20.7 \pm 1.2$ years, $167.0 \pm 5.2 \mathrm{~cm}$, and $68.8 \pm 7.5$ $118 \mathrm{~kg}$, respectively. As shown in Table 1, the participants were divided into two groups: gymnasts $119(\mathrm{GYM} ; \mathrm{N}=16)$ and Judo athletes ( $\mathrm{JD} ; \mathrm{N}=22)$. Judo athletes as well as gymnasts are characterized 120 by a predominant muscular development in the upper limb (Claessens et al. 1991; Ichinose et al. 121 1998; Spenst et al. 1993; Takai et al. 2018). Thus, we adopted judo athletes as a control group. 122 GYM was significantly shorter and lighter than JD. All participants had experienced competitive 123 activities and systematized physical training programs in their major sport for eight or more years. 124 They had competed in intercollegiate or international athletic meetings in the preceding year. The ethical committee of the local university approved this study (the National Institute of Fitness and Sports in Kanoya's Ethics Committee \#11-102). We conducted the study consistent with the requirements for human experimentation in the Declaration of Helsinki. We informed all participants about the purpose and procedures of this study and possible measurements risks before the experiment. All the participants gave their written informed consent for participation in the study.

\section{Experimental design}

In addition to the anthropometric and ultrasound measurements, all participants were involved in maximal voluntary isometric and dynamic contraction tasks. Firstly, anthropometry and ultrasound measurements were conducted. After the standardized warm-up and familiarization with measurement apparatus, the participants were encouraged to perform maximal voluntary isometric contraction (MVC) task, followed by dynamic contraction task, in elbow flexion. After a 5-min rest following the completion the isometric MVC tasks, the dynamic contraction task was conducted. During the tasks, the electromyogram (EMG) activities of elbow flexors and extensors were recorded. All measurements were conducted by the same investigator ( $\mathrm{MN})$.

An earlier finding has demonstrated that the elbow flexion strength is greater in gymnasts than in untrained individuals, but not in elbow extension strength (Niespodzinski et al. 2018). This suggests that gymnastic training would improve the strength capability of the elbow more than that of elbow extensor. Therefore, we examined the F-V relation the elbow flexors in gymnasts.

\section{Measurements of muscle thickness (MT)}

We measured the MTs in the anterior $\left(\mathrm{MT}_{\mathrm{ant}}\right)$ and the posterior $\left(\mathrm{MT}_{\mathrm{pos}}\right)$ part of the upper arm as variables representing the size of elbow flexors and extensors, by using a brightness-mode ultrasound apparatus (ProSound Alpha6, Hitachi Aloka Medical, Japan) with a linear-array probe 
150 (7.27 MHz). The procedure for obtaining ultrasonographic images and for determining MT from 151 the images was identical to that described in an earlier study (Abe et al. 1994). Briefly, the MT 152 measurements for the two sites were conducted at $60 \%$ of the upper arm length defined as the 153 distance from the acromial process to the lateral epicondyle of the humerus. During the measurements, the subjects stood upright with their arms relaxed and extended. The probe was placed perpendicular to the skin without depressing the dermal surface and a probe was coated with water-soluble transmission gel, which provided acoustic contact. The MT was defined as the distance from the subcutaneous adipose tissue-muscle interface to the muscle-bone interface. The upper arm anterior and posterior MTs were referred to as $\mathrm{MT}_{\text {ant }}$ and $\mathrm{MT}_{\text {pos }}$, respectively. The muscles involved in the $\mathrm{MT}_{\text {ant }}$ were the biceps brachii and brachioradialis and that in the $\mathrm{MT}_{\text {pos }}$ was the triceps brachii. All images were analyzed by using image analysis software (Image $\mathrm{J}$ ver. 1.47, NIH, USA). We calculated muscle cross-sectional area index ( $\left.\mathrm{CSA}_{\text {index }}\right)$ of the elbow flexors and extensors by using the following equation (Miyatani et al. 2000):

$$
\mathrm{CSA}_{\text {index }}=\pi \times(\mathrm{MT} / 2)^{2}
$$

where $\pi$ is a constant, 3.14159, and $\mathrm{MT}$ is $\mathrm{MT}_{\text {ant }}$ or $\mathrm{MT}_{\mathrm{pos}}$ in $\mathrm{cm}$. The reproducibility of the MT measurements was assessed on 2 separate days (with an interval of $>4 \mathrm{~d}$ ) in a pilot study with 7 young adults $(25.0 \pm 2.6 \mathrm{yr}, 166.7 \pm 8.7 \mathrm{~cm}$, and $65.0 \pm 7.6 \mathrm{~kg})$. For $\mathrm{MT}_{\text {ant }}$ and $\mathrm{MT}_{\text {pos}}$, there were no significant differences in the mean values between the first and second measurement. The reproducibility of the MT measurements in this study were $1.5-4.1 \%$ for $\mathrm{CV}$ and 0.911 to 0.976 for ICC.

\section{Experimental setup for maximal isometric (MVC) and dynamic contraction tasks}

All the participants performed the MVC and the dynamic contraction elbow flexion tasks with the right arm using a custom-made dynamometer with tension/compression load cells (TR22S, SOHGOH KEISO CO., LTD, Japan) as shown in Figure 1. Participants were seated on an adjustable chair with the shoulder, and hip joints flexed at $90^{\circ}$. Their hips and shoulders were fixed to backrests of chairs, and wrists were fixed to lever arms of the dynamometer in a neutral position by non-elastic belts. The rotation axis of the elbow joint was visually aligned as closely as possible with that of the dynamometer. The forearm was fixed to the lever arm that could rotate freely around the axis with the wrist joint kept in a neutral position. The force signals during the tasks were amplified and attenuated with a low-pass filter ( $<100 \mathrm{~Hz}$, DPM-912B, KYOWA, Japan). The axis of the potentiometer's lever arm was equipped with a dynamometer to detect voltage changes associated with those in the elbow joint angles during the dynamic contraction task. The voltage signals were converted to angle (deg) from the voltage-angle relationship. The force and angle signals were sampled at a frequency of $2 \mathrm{kHz}$ via a 16-bit analog/digital converter (PowerLab/16s: AD Instruments Sydney, Australia) and stored on a personal computer. 


\section{MVC task}

188 Submaximal contractions were conducted as a warm-up exercise. Then, before the dynamic contraction task, the participants conducted the MVC tasks by flexing and extending each elbow joint by gradually exerting elbow flexion or extension force from the baseline to the maximum level, and sustained it at the maximum for approximately $2 \mathrm{~s}$. The elbow joint was held at a $40^{\circ}$ flexed position $\left(0^{\circ}\right.$ corresponds to full elbow extension). After a standardized warm-up protocol $(50 \%$ and $80 \%$ of subjective effect) and familiarization with the measurement apparatus, two trials were performed with a 3-min interval between trials. If the difference between the isometric forces of the two trials was more than $10 \%$, the measurement was made again. The highest value among the 2 or 3 isometric forces was adapted as the elbow flexion $\left(\mathrm{MVF}_{\mathrm{EF}}\right)$ or extension $\left(\mathrm{MVF}_{\mathrm{EE}}\right) \mathrm{MVC}$ force. The $\mathrm{MVF}_{\mathrm{EF}}$ was used to determine the load set in the dynamic contraction task.

\section{Dynamic contraction task}

After a 5-min rest following the completion the MVC tasks, the participants were asked to perform the dynamic contraction task consisting of ballistic contractions against six different loads in a random order (unload condition and 15, 30, 45, 60, 75\% of MVC). They were asked to flex the elbow joint as strongly and quickly as possible in each of the six load conditions. The participants' position and the fixation of the body during the dynamic contraction task were identical to those during the MVC tasks. Weights were attached to pulley moving in conjunction with the lever arm, and the range of the motion was from $40^{\circ}$ to $120^{\circ}$ of the elbow joint angle. A shock absorber was put on the portion at $120^{\circ}$. Before each trial, and an examiner lifted the lever arm until the start position (corresponded to $40^{\circ}$ ) on checking raw data of joint angle with a monitor visually. At the starting position, the participants were kept to relaxed condition by supporting the load by the examiner until the start of elbow flexion with maximal effort. Participants were informed that the magnitude of the load had been set in advance. Rest intervals of $1 \mathrm{~min}$ and $3 \mathrm{~min}$ respectively were set between trials in a given load condition and between loads sets. The analysis of elbow flexion force and velocity at each load condition is described in detail bellows.

\section{Recordings of electromyograms (EMGs)}

Surface EMGs were recorded during the MVC and dynamic contraction tasks from the brachioradialis (Bra), the short head of biceps brachii (BB), and the long head of the triceps brachii (TB) by using bipolar Ag-AgCl electrodes (F-150S, Nihon Koden, Tokyo, Japan) along the direction of the muscle fascicles. Bipolar electrodes $(5 \mathrm{~mm}$ diameter, $20 \mathrm{~mm}$ interelectrode distance) were placed over the muscle bellies after the skin surface was shaved and rubbed with sandpaper and cleaned with alcohol. The electrodes were connected to a differential amplifier 
$222(\times 1000)$ with a bandwidth of 5-1000 Hz. (MEG-6100, Nihon-Kohden, Tokyo, Japan) The EMG 223 signals, as well as force and angle signals, were stored on a personal computer via an analog-to224 digital converter (PowerLab/16s: AD Instruments Sydney, Australia) at a sampling rate of $2 \mathrm{kHz}$. 225 The trial in which the highest MVC force appeared was adopted to analyze the EMG data of every 226 muscle in the MVC task.

We attenuated the EMG amplitude by using a first-order Butterworth high-pass filter (>300 Hz) with a zero-phase lag before rectification, which was following by a first-order Butterworth low-pass filter at $5 \mathrm{~Hz}$ with a zero-phase lag (Yoshitake et al. 2014). We rectified the EMG amplitude during the MVC task and averaged the amplitude over a 1-s window centered at the time when the peak force appeared, which was normalized to this value during the dynamic contraction task. The analysis of the EMG amplitude during the dynamic contraction task is described in detail below.

\section{Velocity, power, and EMG amplitude during dynamic contraction}

Figure 2 shows typical examples of dynamic contraction tasks when unloading, at 30\% and $75 \% \mathrm{MVF}_{\mathrm{EF}}$ in one gymnast. We obtained the angular velocity by differentiating the angle by time. Then, we converted it to the tangential velocity (the elbow flexion velocity, $\mathrm{m} / \mathrm{s}$ ) by multiplying the perpendicular distance between the load cell and the lever-arm axis of the dynamometer. We calculated the power by multiplying the exerted force by the velocity. We averaged each variable over a range of elbow joint angles from $40^{\circ}$ to $100^{\circ}$ and used as functional variables developed for the specific load condition. We referred to the force and velocity as $\mathrm{F}$ and $\mathrm{V}$, respectively, and we obtained the mean power $(\mathrm{P})$ from the product of $\mathrm{F}$ and $\mathrm{V}$. In addition to the absolute values, we expressed $\mathrm{F}$ and $\mathrm{P}$ as values relative to $\mathrm{CSA}_{\text {index }}\left(\mathrm{F} / \mathrm{CSA}_{\text {index }}\right.$ and $\mathrm{P} / \mathrm{CSA}$ index , respectively). The mean values of the filtered EMG for each of the three muscles were expressed as the value relative to the EMG amplitude during the $\mathrm{MVC}$ task $\left(\% \mathrm{EMG}_{\mathrm{MVC}}\right)$.

\section{Calculation of the theoretical maximal force $\left(F_{0}\right)$, velocity $\left(V_{0}\right)$, and power $\left(P_{\max }\right)$}

We calculated the $\mathrm{F}_{0}, \mathrm{~V}_{0}$, and $\mathrm{P}_{\max }$ as basic indicators of the relationship between $\mathrm{F}$ and $\mathrm{V}(\mathrm{F}-\mathrm{V}$ relationship) across the six different loads (Figure 3). We defined the points of intersection of the regression line with the ordinate and transversal axis as $\mathrm{F}_{0}$, and $\mathrm{V}_{0}$, respectively, and calculated $\mathrm{P}_{\max }$ as described in an earlier study (Jaric 2015; Samozino et al. 2012; Vandewalle et al. 1987) by using the following equation:

$$
\mathrm{P}_{\max }=\mathrm{F}_{0} \times \mathrm{V}_{0} / 4 \text {. }
$$

In addition to the absolute values, we expressed $\mathrm{F}_{0}$ and $\mathrm{P}_{\max }$ as values relative to $\mathrm{CSA}_{\text {index }}$ $\left(\mathrm{F}_{0} / \mathrm{CSA}_{\text {index }}\right.$ and $\left.\mathrm{P}_{\text {max }} / \mathrm{CSA}_{\text {index }}\right)$. Furthermore, we adopted the slope of the regression line for the $\mathrm{F}-\mathrm{V}$ relationship ( $\left.\mathrm{F}-\mathrm{V}_{\text {slope }}\right)$ as a parameter indicative of predominance of force (or velocity) in the 
258 relationship (Samozino et al. 2012). To evaluate the test-retest reliability of ballistic power testing, 259 each subject was tested on 2 separate occasions at the same time of day after an interval at least 3 260 days. The same warm-up routine and testing protocol were used in both occasions. To determine 261 the test-retest reliability across the two testing sessions, the intraclass correlation coefficient (ICC ${ }_{1}$, 262 1) was used. There was no significant difference between the two testing sessions in each of $F_{0}, V_{0}$ 263 and $\mathrm{P}_{\max }$. The $\mathrm{ICC}_{(1,1)}$ for each of the measured parameters ranged from 0.820 to 0.984 .

264

Statistics

We have presented descriptive data as means \pm SDs. We used an unpaired Student's t-test to examine differences in measured variables between GYM and JD, and a two-way repeated measures analysis of variance (ANOVA: 2 groups $\times 6$ loads) to test the main effects of group and load and their interaction on $\% \mathrm{EMG}_{\mathrm{MVC}}$ for the examined muscles. When appropriate, we used simple main effect test was used to test the significance of the group difference for post hoc comparison. We calculated Pearson's product-moment correlation coefficient $(r)$ to examine the associations between $\mathrm{F}$ and V. We also calculated Cohen's $\mathrm{d}$ (for a post hoc test) and $\eta^{2}$ (for ANOVA) as indices of effect sizes. We interpreted Cohen's $d$ as large: $\geq 0.80$, medium: 0.50-0.79, small: 0.20-0.49, or trivial: $<0.20$, and we interpreted $\eta^{2}$ was as large: 0.14, medium: 0.06, or small: 0.01 (Cohen 1988). Sphericity was checked by Mauchly's test in ANOVA, and $P$ values were modified with Greenhouse-Geisser correction when necessary. We set the level of significance as $p<0.05$. We analyzed all the data using SPSS software (SPSS statistics 25; IBM, Japan). 
279

280

281

282

283

284

285

286

287

288

289

290

291

292

293

294

295

296

297

298

299

300

301

302

303

304

305

306

307

308

\section{Results}

There were no significant differences in $\mathrm{MT}_{\text {ant }}$ and $\mathrm{CSA}_{\text {index }}$ of elbow flexor between GYM and JD, although MTpos and CSAindex of elbow extensor were significantly smaller in GYM than in JD (Table 1). $\mathrm{MVF}_{\mathrm{EF}} / \mathrm{CSA}_{\text {index }}$ for GYM was significantly lower than that for JD, while the corresponding difference was not found in $\mathrm{MVF}_{\mathrm{EE}} / \mathrm{CSA}_{\text {index }}$.

$* * *$ Table 1

Figure 3 shows an example of F-V relationship. $\mathrm{F}$ was linearly associated with $\mathrm{V}$ in all the participants ( $r=-0.997$ to -0.905 for GYM, $r=-0.998$ to -0.840 for JD). Each of the theoretical maximum parameters was significantly lower in GYM than in JD (Table 2). In addition, the F$\mathrm{V}_{\text {slope }}$ was steeper in GYM compared to JD. The $\mathrm{F}_{0} / \mathrm{CSA}_{\text {index }}$ and $\mathrm{P}_{\max } / \mathrm{CSA}_{\text {index }}$ were significantly lower in GYM than in JD (Table 2).

*** Figure 3

A two-way ANOVA indicated neither a significant interaction between $\% \mathrm{EMG}_{\mathrm{MVC}}$ and load nor a significant main effect of group for $\operatorname{Bra}\left(p=0.173, \eta^{2}=0.206\right)$ and $\operatorname{TB}\left(p=0.563, \eta^{2}=\right.$ 0.481): $125.9 \pm 49.2 \%$ for Bra, and $6.8 \pm 2.5 \%$ for TB in GYM and $120.1 \pm 32.6 \%$ for Bra, and $9.7 \pm 8.3 \%$ for TB in JD. For BB, however, the ANOVA revealed a significant interaction $(p=$ $\left.0.017, \eta^{2}=0.080\right)$. The $\% \mathrm{EMG}_{\mathrm{MVC}}$ of BB at unload condition was lower in GYM than in JD ( $p=$ 0.022 , Cohen's $d=1.41$ ). In addition, the $\% \mathrm{EMG}_{\mathrm{MVC}}$ values of $\mathrm{BB}$ at 30 and $40 \% \mathrm{MVC}$ conditions tended to be lower in GYM compared to JD ( $p=0.069-0.083$, Cohen's $\mathrm{d}=0.663-0.923)$.

$* * *$ Table 2

$\mathrm{F}_{0}, \mathrm{~V}_{0}, \mathrm{P}_{\max }$ and $\mathrm{F}-\mathrm{V}_{\text {slope }}$ were significantly lower in GYM $(260.9 \pm 47.1 \mathrm{~N}, 1.5 \pm 0.4$ $\mathrm{m} / \mathrm{s}, 96.3 \pm 23.9 \mathrm{~W},-190.5 \pm 91.2)$ than in JD $(311.5 \pm 63.0 \mathrm{~N}, 2.2 \pm 0.3 \mathrm{~m} / \mathrm{s}, 173.2 \pm 41.6 \mathrm{~W}$, $143.3 \pm 39.1)$. 
309

310

311

312

\section{Discussion}

The main findings obtained here were that 1) GYM had lower $F_{0}, V_{0}, P_{\max }$, and F- $\mathrm{V}_{\text {slope }}$ than JD, 2) GYM had lower $\mathrm{MVF}_{\mathrm{EF}} / \mathrm{CSA}_{\text {index }}$ and $\mathrm{F}_{0} / \mathrm{CSA}_{\text {index }}$ than JD, and 3) the activity levels of $\mathrm{BB}$ during the dynamic tasks tended to be lower in GYM than in JD at load of $<45 \% \mathrm{MVC}$. The regression line slope of the F-V relationship in athletes reflects their competitive and training activity profiles, and it becomes a parameter for discriminating force- or velocity-oriented type of athletes (Bozic \& Bacvarevic 2018; Giroux et al. 2016; Izquierdo et al. 2002; McBride et al. 1999). Thus, the result on F- $\mathrm{V}_{\text {slope }}$ indicates that as compared to JD, gymnasts show a force-orientated profile in explosive elbow flexion. Furthermore, the second result supports the hypothesis that the F-V relationship of elbow flexors in gymnasts is characterized by the low capacity for generating an explosive force relative to muscle size. In addition. The third result implies that the observed force-orientated profile and low $\mathrm{V}_{0}, \mathrm{~F}_{0} / \mathrm{CSA}_{\text {index }}$, and $\mathrm{P}_{\max }$ in GYM might be partially attributable to low activation of elbow flexors during explosive dynamic contractions in this population, notably in conditions requiring quick contraction against light loads.

There are three possible explanations for the force-oriented profile and the lower power generating capacity in GYM compared to JD. (1) An imbalance between morphological adaptation and neural adaptation of the elbow flexors caused by long-term gymnastic training; (2) lower muscular activation during explosive elbow flexion; and (3) increased hypertrophied muscles relative to limb length. Firstly, as described earlier, the activities of upper limb muscles during gymnastics can be characterized by highly intense and sustained contractions and/or cocontractions between the agonist and antagonistic muscles. Prolonged maximum voluntarily cocontraction training produces a significant gain in muscle size without an improvement in muscle strength (Maeo et al. 2014). Mitchell et al. (2012) have proposed that training-induced gains in the muscle volume of the quadriceps femoris were similar between training programs with $30 \%$ and $80 \%$ of $1 \mathrm{RM}$ to failure, but isotonic maximal strength gain was more significant in high-intensity than in low-intensity programs. These findings suggest that a training modality with long-term sustained contractions would result in an imbalance between hypertrophic and neuromuscular adaptations of exercising muscles. Furthermore, Kochanowicz et al. (2018b) reported no significant difference in elbow flexion strength between gymnasts and untrained individuals, whereas gymnasts had a greater lean tissue mass in the arms than untrained individuals. Crosssectional studies have also provided evidence that dynamic strength normalized to the muscle size of body-builders, who are generally categorized as the practitioners of high-volume resistance exercises (Hackett et al. 2013), is lower at the whole muscle (Alway et al. 1990; Sale et al. 1987) and single muscle fiber (Meijer et al. 2015) levels than in non-athletes or power athletes. Taken together, it is likely that long-term participation in gymnastics training produces a relatively higher muscle size gain than isometric or dynamic strength, and consequently causes the low $\mathrm{F}_{0} / \mathrm{CSA}_{\text {index }}$ 
345

346

347

348

349

350

351

352

353

354

355

356

357

358

359

360

361

362

363

364

365

366

367

368

369

370

371

372

373

374

375

376

377

378

379

380

in gymnasts, i.e., muscle quality.

Secondly, the muscular activities of BB during explosive elbow flexion at relatively low load tended to be lower in GYM than in JD, whereas no significant group difference in submaximal EMG amplitude during isometric contraction was found in this study (Supplemental data). Combined this with the current finding, the lower muscular activities during dynamic contraction task in GYM may be explained as a result of sport-specific adaptation in the BB of this athletic group. Agonist muscle activation in the early phase of explosive torque development is strongly associated with the initial torque output in isometric knee extension contractions (de Ruiter et al. 2004; de Ruiter et al. 2006; de Ruiter et al. 2007). Highly intense and sustained training elicits muscle hypertrophy (Massey et al. 2018) and attenuates the activation level in the earlier phases of force development during explosive isometric knee extensions (Balshaw et al. 2016; Tillin \& Folland 2014). Furthermore, training modalities with slow movements and tonic force generation that causes sustained muscular activity increases isometric strength and muscle size (Tanimoto \& Ishii 2006), but has little effect on dynamic strength and power production (Tanimoto \& Ishii 2006; Usui et al. 2016). Considering these findings, lower muscular activation level of BB during explosive elbow flexion in gymnasts might be due to type of training modality in gymnasts.

Thirdly, GYM had higher ratios of $\mathrm{CSA}_{\text {index }}$ and $\mathrm{MT}_{\text {ant }}$ to upper arm length: $0.34 \pm 0.06$ $\mathrm{cm}^{2} / \mathrm{cm}$ for GYM vs. $0.30 \pm 0.06 \mathrm{~cm}^{2} / \mathrm{cm}$ for JD in $\mathrm{CSA}_{\mathrm{EF}}(p=0.032$, Cohen's $\mathrm{d}=0.73)$ and 0.12 $\pm 0.01 \mathrm{~cm} / \mathrm{cm}$ for GYM vs. $0.11 \pm 0.01 \mathrm{~cm} / \mathrm{cm}$ for JD in $\mathrm{MT}_{\text {ant }}(p=0.003$, Cohen's $\mathrm{d}=1.05)$. The mean values of the ratio of $\mathrm{MT}_{\text {ant }}$ to upper arm length in GYM and JD were higher by $18 \%$ and $5 \%$, respectively, compared to reference data obtained from the general Japanese population (Wakahara et al. 2010), which indicates that GYM has a larger elbow flexor muscle size for a given upper arm length. Most fibers of elbow flexors have equal length and uniform thickness (Kaufman et al. 1989). The fibers in this muscle group are attached to a tendon plate that extends into the muscle belly and organizes a large number of fibers with similar length and thickness in parallel, which is called the "parallelepipedon" (An et al. 1981). When a muscle is hypertrophied, the length of the tendon plate appears to be extended further into the muscle belly, and the fibers must pull at a more oblique angle to the direction of induced motion (the line of pull of the tendon end) (Kaufman et al. 1989). Therefore, the fiber alignment is more oblique to the force loss in the line of action. The influence of this could be greater at higher contraction velocities (Maughan et al. 1984). Therefore, the low $\mathrm{F}_{0} / \mathrm{CSA}_{\text {index }}$ in GYM might be caused by the morphological profile of elbow flexor muscles that is characterized by a high ratio of muscle size to upper limb length.

In addition to the aforementioned aspects, the influence of fiber composition might also be involved to explain why GYM showed lower $\mathrm{F}_{0}, \mathrm{~V}_{0}$ and $\mathrm{P}_{\max }$ than JD. It is known that a 14week resistance training of the quadriceps femoris yields a reduction in the relative portion of type IIX muscle fiber, and its decline negatively influenced the rate of force development in the early 
381

382

phase (<100 ms) (Andersen et al. 2010). Furthermore, Kesidis et al. (2008) observed lower percentage of type IIX fiber for the vastus lateralis in bodybuilders than in physical education students. If these findings can be applied to the current results, there is a possibility that low $\mathrm{V}_{0}$ in GYM compared to JD might be due to the group difference in the percentage of type IIX fiber.

There are some limitations in this study. Firstly, we determined MT as a measure of muscle size and used $\mathrm{CSA}_{\text {index }}$ calculated from MT to normalize F. Miyatani et al. (2000) reported that the sum of the product of $\mathrm{CSA}_{\text {index }}$ and upper arm length for the elbow flexors and extensors strongly correlated with the MRI-based muscle volumes of the two muscle groups $(r=0.962)$. These findings indicate that either MT or $\mathrm{CSA}_{\text {index }}$ adopted here can be qualitative parameters of a specific muscle group, although the previous studies have not examined the direct associations of these variables with the muscle CSA of the elbow flexors. At the same time, the reports of Miyatani et al. (2000) warrants to interpret the current results as that the muscle quality of elbow flexors in GYM is lower than that in JD. Secondly, the muscle activities during handstand are higher in the elbow extensors than in the elbow flexors (Kochanowicz et al. 2018a). Furthermore, F-V profile may be affected by muscle architecture (Morales-Artacho et al. 2018). The elbow flexors are mainly consisted of parallel muscles and the elbow extensors are pennate muscles. Therefore, the F-V profile of the elbow extensors would be different from that of the elbow flexors. Thirdly, it is known that force-velocity profile of the upper body differs between men and women (Torrejón et al. 2019). We have no data concerning the force-velocity profile of female gymnasts. Hence, we cannot conclude whether the current findings are applied to female gymnasts. Further investigations are needed to clarify these points. 


\section{Practical application}

404 The current findings indicate that gymnasts cannot generate explosive elbow flexion force 405 corresponding to their muscle size. This may be due to low neuromuscular activities during the 406 maximal dynamic tasks against relatively low loads. As described earlier, gymnasts are 407 frequently required to support their body mass and control body balance by using the upper 408 extremities while overcoming repetitive high-impact loadings (DiFiori et al. 2002). This implies 409 that regardless of elbow flexors and extensors, to gain the explosive force generation capability 410 of the upper limb muscles will be a factor for improving gymnastic performance. Training411 induced changes in muscle functions and activation in the early phase of force development 412 depend on the type of muscle contraction (sustained vs. explosive) (Balshaw et al. 2016; Massey 413 et al. 2018; Tillin \& Folland 2014), load adapted, and contraction velocities (Kaneko et al. 414 1984). Ballistic and/or explosive exercises can greatly improve power production (Cormie et al. 415 2011). On the other hand, a training modality with intense and sustained muscle contractions is 416 less effective for explosive muscle functions and activation compared to that consisting of 417 explosive exercise (Balshaw et al. 2016). Taking these aspects into account together with the 418 findings obtained here, it will be recommended for gymnasts and their coaches that for 419 improving explosive force generation capacity of the elbow flexors, training program including 420 ballistic and/or explosive exercises for this muscle group should be involved to the schedule of 421 their regular training activities. 


\section{Conclusions}

424 The current findings demonstrate that as compared to judo athletes, gymnasts have a force-oriented 425 profile and low capacity for generating explosive force in elbow flexors, which is partially due to 426 neuromuscular activity during explosive elbow flexion against relatively low load and force 427 exerted normalized to muscle size.

428 


\section{Acknowledgments}

430 The authors wish to express their gratitude to the students of the National Institute of Fitness and 431 Sports in Kanoya for their contribution to this study. This study was supported by a NIFS project 432 for the assessment of physical fitness for athletes.

433 
434

\section{References}

Abe T, Kondo M, Kawakami Y, and Fukunaga T. 1994. Prediction equations for body composition of Japanese adults by B-mode ultrasound. American Journal of Human Biology 6:161-170. 10.1002/ajhb.1310060204

Alway SE, Stray-Gundersen J, Grumbt WH, and Gonyea WJ. 1990. Muscle cross-sectional area and torque in resistance-trained subjects. European Journal of Applied Physiology and Occupational Physiology 60:86-90.

An KN, Hui FC, Morrey BF, Linscheid RL, and Chao EY. 1981. Muscles across the elbow joint: a biomechanical analysis. Journal of Biomechanics 14:659-669.

Andersen LL, Andersen JL, Zebis MK, and Aagaard P. 2010. Early and late rate of force development: differential adaptive responses to resistance training? Scandinavian Journal of Medicine \& Science in Sports 20:e162-169. 10.1111/j.1600-0838.2009.00933.x

Asci A, and Acikada C. 2007. Power production among different sports with similar maximum strength. Journal of Strength and Conditioning Research 21:10-16. 10.1519/r-16474.1

Baker D. 2001. Comparison of upper-body strength and power between professional and college-aged rugby league players. Journal of Strength and Conditioning Research $15: 30-35$.

Baker DG, and Newton RU. 2006. Adaptations in upper-body maximal strength and power output resulting from long-term resistance training in experienced strength-power athletes. Journal of Strength and Conditioning Research 20:541-546. 10.1519/r-16024.1

Balshaw TG, Massey GJ, Maden-Wilkinson TM, Tillin NA, and Folland JP. 2016. Trainingspecific functional, neural, and hypertrophic adaptations to explosive- vs. sustainedcontraction strength training. Journal of Applied Physiology 120:1364-1373. 10.1152/japplphysiol.00091.2016

Bernasconi SM, Tordi NR, Parratte BM, and Rouillon JD. 2009. Can shoulder muscle coordination during the support scale at ring height be replicated during training exercises in gymnastics? Journal of Strength and Conditioning Research 23:2381-2388. 10.1519/JSC.0b013e3181bac69f

Bozic P, and Bacvarevic B. 2018. Force velocity profiles of elite athletes tested on a cycle ergometer. Montenegrin Journal of Sports Science \& Medicine 7:2381-2388.

Claessens AL, Veer FM, Stijnen V, Lefevre J, Maes H, Steens G, and Beunen G. 1991. Anthropometric characteristics of outstanding male and female gymnasts. Journal of Sports Sciences 9:53-74. 10.1080/02640419108729855

Cohen J. 1988. Statistical power analysis for the behavioral sciences. Lawrence Erlbaum.

Cormie P, McGuigan MR, and Newton RU. 2011. Developing maximal neuromuscular power: part 2 - training considerations for improving maximal power production. Sports 
Medicine 41:125-146. 10.2165/11538500-000000000-00000

Daly R, Rich P, Klein R, and Bass S. 1999. Effects of high-impact exercise on ultrasonic and biomechanical indices of skeletal status: a prospective study in young male gymnasts. Journal of Bone and Mineral Research 14:1222-1230.

de Ruiter CJ, Kooistra RD, Paalman MI, and de Haan A. 2004. Initial phase of maximal voluntary and electrically stimulated knee extension torque development at different knee angles. Journal of Applied Physiology 97:1693-1701. 10.1152/japplphysiol.00230.2004

de Ruiter CJ, Van Leeuwen D, Heijblom A, Bobbert MF, and de Haan A. 2006. Fast unilateral isometric knee extension torque development and bilateral jump height. Medicine and Science in Sports and Exercise 38:1843-1852. 10.1249/01.mss.0000227644.14102.50

de Ruiter CJ, Vermeulen G, Toussaint HM, and de Haan A. 2007. Isometric knee-extensor torque development and jump height in volleyball players. Medicine and Science in Sports and Exercise 39:1336-1346. 10.1097/mss.0b013e318063c719

DiFiori JP, Puffer JC, Aish B, and Dorey F. 2002. Wrist pain, distal radial physeal injury, and ulnar variance in young gymnasts: does a relationship exist? The American Journal of Sports Medicine 30:879-885. 10.1177/03635465020300062001

Djuric S, Cuk I, Sreckovic S, Mirkov D, Nedeljkovic A, and Jaric S. 2016. Selective Effects of Training Against Weight and Inertia on Muscle Mechanical Properties. International Journal of Sports Physiology and Performance 11:927-932. 10.1123/ijspp.2015-0527

Fukunaga T, Miyatani M, Tachi M, Kouzaki M, Kawakami Y, and Kanehisa H. 2001. Muscle volume is a major determinant of joint torque in humans. Acta Physiologica Scandinavica 172:249-255. 10.1046/j.1365-201x.2001.00867.x

Giroux C, Rabita G, Chollet D, and Guilhem G. 2016. Optimal Balance Between Force and Velocity Differs Among World-Class Athletes. Journal of Applied Biomechanics 32:5968. 10.1123/jab.2015-0070

Hackett DA, Johnson NA, and Chow CM. 2013. Training practices and ergogenic aids used by male bodybuilders. Journal of Strength and Conditioning Research 27:1609-1617. 10.1519/JSC.0b013e318271272a

Ichinose Y, Kanehisa H, Ito M, Kawakami Y, and Fukunaga T. 1998. Relationship between muscle fiber pennation and force generation capability in Olympic athletes. International journal of Sports Medicine 19:541-546. 10.1055/s-2007-971957

Izquierdo M, Hakkinen K, Gonzalez-Badillo JJ, Ibanez J, and Gorostiaga EM. 2002. Effects of long-term training specificity on maximal strength and power of the upper and lower extremities in athletes from different sports. European Journal of Applied Physiology 87:264-271. 10.1007/s00421-002-0628-y 
Jaric S. 2015. Force-velocity Relationship of Muscles Performing Multi-joint Maximum Performance Tasks. International Journal of Sports Medicine 36:699-704. 10.1055/s0035-1547283

Jimenez-Reyes P, Samozino P, Brughelli M, and Morin JB. 2016. Effectiveness of an Individualized Training Based on Force-Velocity Profiling during Jumping. Frontiers in Physiology 7:677. 10.3389/fphys.2016.00677

Kaneko M, Fuchimoto T, Toji H, and Suei K. 1984. Training effect of different loads on the force-velocity relationship and mechanical power output in human muscle. Scandinavian Journal of Sports Sciences 5:50-55.

Kaufman KR, An KN, and Chao EY. 1989. Incorporation of muscle architecture into the muscle length-tension relationship. Journal of Biomechanics 22:943-948.

Kesidis N, Metaxas TI, Vrabas IS, Stefanidis P, Vamvakoudis E, Christoulas K, Mandroukas A, Balasas D, and Mandroukas K. 2008. Myosin heavy chain isoform distribution in single fibres of bodybuilders. European Journal of Applied Physiology 103:579-583. 10.1007/s00421-008-0751-5

Kochanowicz A, Niespodziński B, Marina M, Mieszkowski J, Biskup L, and Kochanowicz K. 2018a. Relationship between postural control and muscle activity during a handstand in young and adult gymnasts. Human Movement Science 58:195-204. 10.1016/j.humov.2018.02.007

Kochanowicz A, Niespodzinski B, Mieszkowski J, Kochanowicz K, and Sawczyn S. 2018b. The effect of gymnastic training on muscle strength and co-activation during isometric elbow and glenohumeral flexion/extension. The Journal of Sports Medicine and Physical Fitness 58:966-973. 10.23736/s0022-4707.17.06916-x

Kochanowicz A, Niespodziński B, Mieszkowski J, Sawczyn S, Cięszczyk P, and Kochanowicz K. 2019. Neuromuscular and Torque Kinetic Changes After 10 Months of Explosive Sport Training in Prepubertal Gymnasts. Pediatric Exercise Science 31:77-84. 10.1123/pes.2018-0034

Maeo S, Yoshitake Y, Takai Y, Fukunaga T, and Kanehisa H. 2014. Neuromuscular adaptations following 12-week maximal voluntary co-contraction training. European Journal of Applied Physiology 114:663-673. 10.1007/s00421-013-2801-x

Massey GJ, Balshaw TG, Maden-Wilkinson TM, Tillin NA, and Folland JP. 2018. Tendinous Tissue Adaptation to Explosive- vs. Sustained-Contraction Strength Training. Frontiers in Physiology 9:1170. 10.3389/fphys.2018.01170

Maughan RJ, Watson JS, and Weir J. 1984. Muscle strength and cross-sectional area in man: a comparison of strength-trained and untrained subjects. British Journal of Sports Medicine 18:149-157. 10.1136/bjsm.18.3.149 
McBride J, Triplett-McBride T, Davie A, and Newton R. 1999. A comparison of strength and power characteristics between power lifters, Olympic lifters, and sprinters. Journal of Strength and Conditioning Research 13:58-66.

Meijer JP, Jaspers RT, Rittweger J, Seynnes OR, Kamandulis S, Brazaitis M, Skurvydas A, Pisot R, Simunic B, Narici MV, and Degens H. 2015. Single muscle fibre contractile properties differ between body-builders, power athletes and control subjects. Experimental Physiology 100:1331-1341. 10.1113/ep085267

Mitchell CJ, Churchward-Venne TA, West DW, Burd NA, Breen L, Baker SK, and Phillips SM. 2012. Resistance exercise load does not determine training-mediated hypertrophic gains in young men. Journal of Applied Physiology 113:71-77. 10.1152/japplphysiol.00307.2012

Miyatani M, Kanehisa H, and Fukunaga T. 2000. Validity of bioelectrical impedance and ultrasonographic methods for estimating the muscle volume of the upper arm. European Journal of Applied Physiology 82:391-396. 10.1007/s004210000213

Morales-Artacho AJ, Ramos AG, Pérez-Castilla A, Padial P, Argüelles-Cienfuegos J, de la Fuente B, and Feriche B. 2018. Associations of the Force-velocity Profile with Isometric Strength and Neuromuscular Factors. International Journal of Sports Medicine 39:984-994. 10.1055/a-0644-3742

Niespodzinski B, Kochanowicz A, Mieszkowski J, Piskorska E, and Zychowska M. 2018. Relationship between Joint Position Sense, Force Sense, and Muscle Strength and the Impact of Gymnastic Training on Proprioception. BioMed Research International 2018:5353242. 10.1155/2018/5353242

Sale DG, MacDougall JD, Alway SE, and Sutton JR. 1987. Voluntary strength and muscle characteristics in untrained men and women and male bodybuilders. Journal of Applied Physiology 62:1786-1793. 10.1152/jappl.1987.62.5.1786

Samozino P, Rejc E, Di Prampero PE, Belli A, and Morin JB. 2012. Optimal force-velocity profile in ballistic movements--altius: citius or fortius? Medicine and Science in Sports and Exercise 44:313-322. 10.1249/MSS.0b013e31822d757a

Spenst LF, Martin AD, and Drinkwater DT. 1993. Muscle mass of competitive male athletes. Journal of Sports Sciences 11:3-8. 10.1080/02640419308729956

Takai Y, Nakatani M, Aoki T, Komori D, Oyamada K, Murata K, Fujita E, Akamine T, Urita Y, Yamamoto M, and Kanehisa H. 2018. Body shape indices are predictors for estimating fat-free mass in male athletes. PLoS One 13:e189836. 10.1371/journal.pone.0189836

Tanimoto M, and Ishii N. 2006. Effects of low-intensity resistance exercise with slow movement and tonic force generation on muscular function in young men. Journal of 
Applied Physiology 100:1150-1157. 10.1152/japplphysiol.00741.2005

Tillin NA, and Folland JP. 2014. Maximal and explosive strength training elicit distinct neuromuscular adaptations, specific to the training stimulus. European Journal of Applied Physiology 114:365-374. 10.1007/s00421-013-2781-x

Torrejón A, Balsalobre-Fernández C, Haff GG, and García-Ramos A. 2019. The loadvelocity profile differs more between men and women than between individuals with different strength levels. Sports Biomechanics 18:245-255. 10.1080/14763141.2018.1433872

Usui S, Maeo S, Tayashiki K, Nakatani M, and Kanehisa H. 2016. Low-load Slow Movement Squat Training Increases Muscle Size and Strength but Not Power. International Journal of Sports Medicine 37:305-312. 10.1055/s-0035-1564255

Vandewalle H, Peres G, Heller J, Panel J, and Monod H. 1987. Force-velocity relationship and maximal power on a cycle ergometer. Correlation with the height of a vertical jump. European Journal of Applied Physiology and Occupational Physiology 56:650-656.

Vuk S, Markovic G, and Jaric S. 2012. External loading and maximum dynamic output in vertical jumping: the role of training history. Human Movement Science 31:139-151. 10.1016/j.humov.2011.04.007

Wakahara T, Kanehisa H, Kawakami Y, Fukunaga T, and Yanai T. 2013. Relationship between muscle architecture and joint performance during concentric contractions in humans. Journal of Applied Biomechanics 29:405-412.

\section{Wakahara T, Takeshita K, Kato E, Miyatani M, Tanaka NI, Kanehisa H, Kawakami Y,} and Fukunaga T. 2010. Variability of limb muscle size in young men. American Journal of Human Biology 22:55-59. 10.1002/ajhb.20951

Yoshitake Y, Takai Y, Kanehisa H, and Shinohara M. 2014. Muscle shear modulus measured with ultrasound shear-wave elastography across a wide range of contraction intensity. Muscle \& Nerve 50:103-113. 10.1002/mus.24104 


\section{Table 1 (on next page)}

Table 1. Physical characteristics of the participants 
Table 1. Physical characteristics of the participants

\begin{tabular}{|c|c|c|c|c|c|c|c|c|}
\hline \multirow{2}{*}{$\begin{array}{l}\text { Variables } \\
\text { Height, cm }\end{array}$} & \multicolumn{3}{|c|}{ GYM, $n=16$} & \multicolumn{3}{|c|}{$\mathbf{J D}, \mathbf{n}=\mathbf{2 2}$} & \multirow{2}{*}{$\begin{array}{c}\boldsymbol{p} \\
<0.001\end{array}$} & \multirow{2}{*}{$\begin{array}{c}\text { Cohen's } \boldsymbol{d} \\
1.47\end{array}$} \\
\hline & 163.0 & \pm & 4.0 & 170.9 & \pm & 6.5 & & \\
\hline Body mass, $\mathrm{kg}$ & 58.9 & \pm & 2.8 & 78.8 & \pm & 12.2 & $<0.001$ & 2.24 \\
\hline Upper arm length, cm & 30.6 & \pm & 1.3 & 32.4 & \pm & 1.8 & 0.001 & 1.17 \\
\hline $\mathrm{MT}_{\mathrm{ant}}, \mathrm{cm}$ & 3.6 & \pm & 0.3 & 3.5 & \pm & 0.4 & 0.212 & 0.43 \\
\hline $\mathrm{MT}_{\text {pos }}, \mathrm{cm}$ & 4.3 & \pm & 0.5 & 4.8 & \pm & 0.4 & 0.005 & 0.98 \\
\hline $\mathrm{CSA}_{\text {index }}$ of elbow flexor & 10.4 & \pm & 1.6 & 9.6 & \pm & 2.2 & 0.255 & 0.39 \\
\hline $\mathrm{CSA}_{\text {index }}$ of elbow extensor & 15.0 & \pm & 2.9 & 18.1 & \pm & 3.4 & 0.005 & 0.99 \\
\hline $\mathrm{MVF}_{\mathrm{EF}}, \mathrm{N}$ & 242.5 & \pm & 23.6 & 284.8 & \pm & 45.8 & 0.001 & 1.16 \\
\hline $\mathrm{MVF}_{\mathrm{EE}}, \mathrm{N}$ & 201.0 & \pm & 47.8 & 262.9 & \pm & 79.2 & 0.005 & 0.95 \\
\hline $\mathrm{MVF}_{\mathrm{EF}} / \mathrm{CSA}_{\text {index }}, \mathrm{N} / \mathrm{cm}^{2}$ & 23.7 & \pm & 3.0 & 30.4 & \pm & 5.3 & $<0.001$ & 1.52 \\
\hline $\mathrm{MVF}_{\mathrm{EE}} / \mathrm{CSA}_{\text {index }}, \mathrm{N} / \mathrm{cm}^{2}$ & 13.7 & \pm & 2.9 & 14.8 & \pm & 4.9 & 0.411 & 0.28 \\
\hline
\end{tabular}

2 Values are means \pm SDs.

$3 \mathrm{MT}_{\mathrm{ant}}$, muscle thickness at upper arm anterior

$4 \mathrm{MT}_{\text {pos }}$, muscle thickness at upper arm posterior

$5 \mathrm{CSA}_{\text {index }}$, muscle cross-sectional area index obtained using the equation of $\pi \times(\mathrm{MT} / 2)^{2}$

$6 \quad \mathrm{MVF}_{\mathrm{EF}}$, maximal voluntary isometric elbow flexion force

$7 \mathrm{MVF}_{\mathrm{EE}}$, maximal voluntary isometric elbow extension force 


\section{Table 2 (on next page)}

Table 2. Descriptive data on the parameters derived from force-velocity relation of elbow flexors 
Table 2. Descriptive data on the parameters derived from force-velocity relation of elbow flexors

\begin{tabular}{|c|c|c|c|c|c|c|c|c|}
\hline \multirow{2}{*}{$\begin{array}{l}\text { Variables } \\
\mathrm{F}_{0}, \mathrm{~N}\end{array}$} & \multicolumn{3}{|c|}{ GYM, $n=16$} & \multicolumn{3}{|c|}{$\mathrm{JD}, \mathbf{n}=\mathbf{2 2}$} & \multirow{2}{*}{$\begin{array}{c}\boldsymbol{p} \\
0.010\end{array}$} & \multirow{2}{*}{$\begin{array}{c}\text { Cohen's } \\
\boldsymbol{d} \\
0.89\end{array}$} \\
\hline & 260.9 & \pm & 47.1 & 311.5 & \pm & 63.0 & & \\
\hline $\mathrm{V}_{0}, \mathrm{~m} / \mathrm{s}$ & 1.5 & \pm & 0.4 & 2.2 & \pm & 0.3 & $<0.001$ & 2.11 \\
\hline $\mathrm{P}_{\max }, \mathrm{W}$ & 96.3 & \pm & 23.9 & 173.2 & \pm & 41.6 & $<0.001$ & 2.17 \\
\hline $\mathrm{F}-\mathrm{V}_{\text {slope }}$ & -190.5 & \pm & 91.2 & -143.3 & \pm & 39.1 & 0.036 & 0.72 \\
\hline $\mathrm{F}_{0} / \mathrm{CSA}_{\text {index }}, \mathrm{N} / \mathrm{cm}^{2}$ & 25.3 & \pm & 3.6 & 33.0 & \pm & 5.8 & $<0.001$ & 1.54 \\
\hline $\mathrm{P}_{\max } / \mathrm{CSA}_{\text {index }}, \mathrm{W} / \mathrm{cm}^{2}$ & 9.4 & \pm & 2.4 & 18.3 & \pm & 3.9 & $<0.001$ & 2.63 \\
\hline
\end{tabular}

2 Values are means \pm SDs.

$3 \quad \mathrm{~F}_{0}$, theoretical maximal force

$4 \quad \mathrm{~V}_{0}$, theoretical maximal velocity

$5 \mathrm{P}_{\max }$, theoretical maximal power

$6 \quad \mathrm{~F}-\mathrm{V}_{\text {slope }}$, slope of the regression line for the relationship between force and velocity

$7 \mathrm{CSA}_{\text {index }}$, muscle cross-sectional area index obtained using the equation of $\pi \times(\mathrm{MT} / 2)^{2}$ 
Figure 1

Experimental setup for maximal isometric (MVC) and dynamic contraction tasks.

Schematic diagram of the experimental set up for conducting the maximal isometric (MVC) and dynamic contraction tasks. The participants sat on a chair adjusted for the testing position. Their right arms were fixed to the dynamometer with the shoulder flexed at $90^{\circ}$ and the forearm in a neutral position.

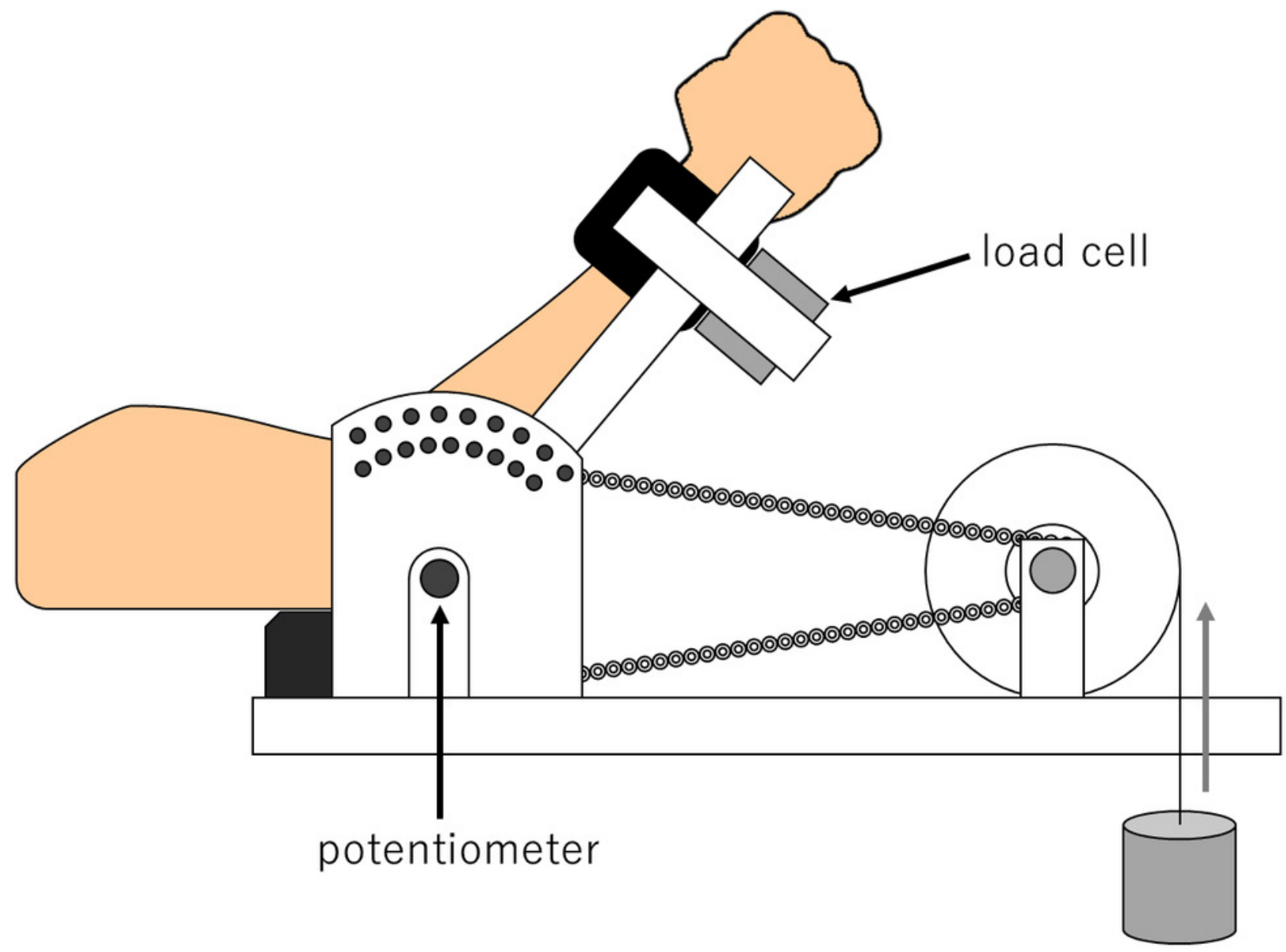


Figure 2

Typical examples of dynamic contraction tasks.

Typical examples of the elbow joint angle (A), force (B), velocity (C), power (D), and the EMG amplitude of $\mathrm{BB}(\mathrm{E})$ during the dynamic contraction task when unloading, $30 \%$ and $75 \% \mathrm{MVF}_{\mathrm{EF}}$ for one gymnast. 

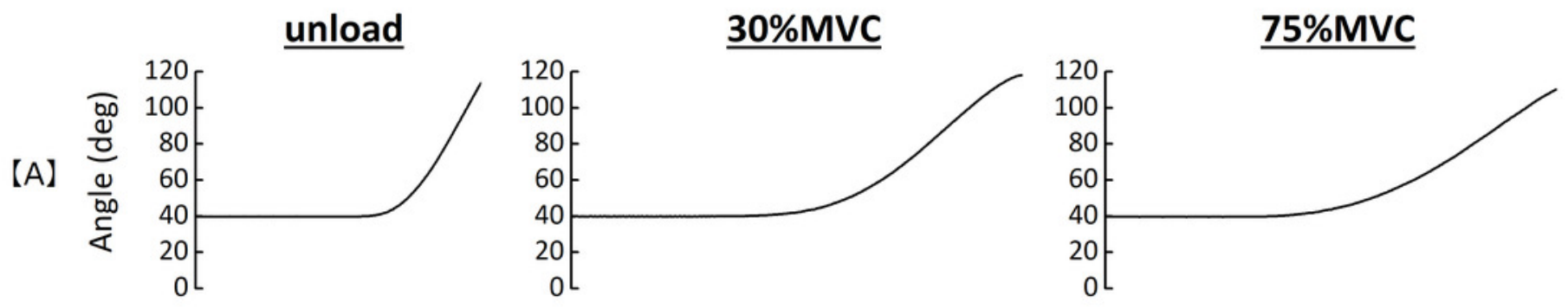

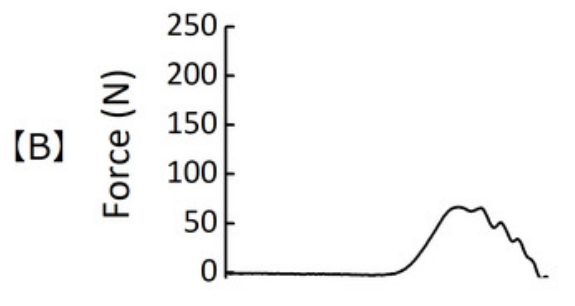

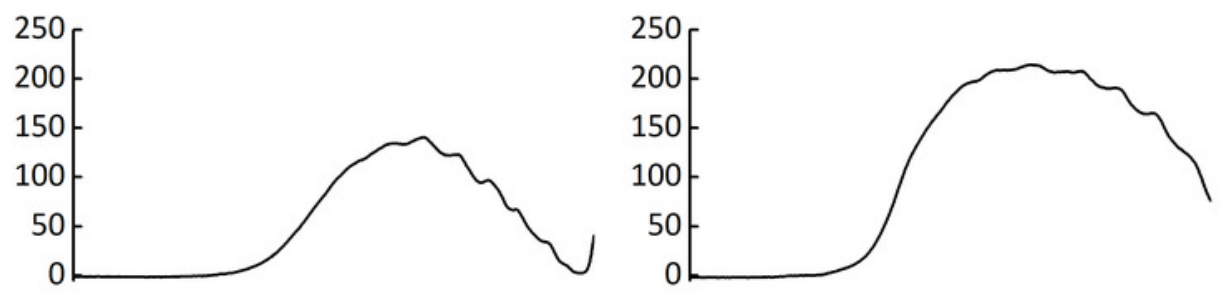

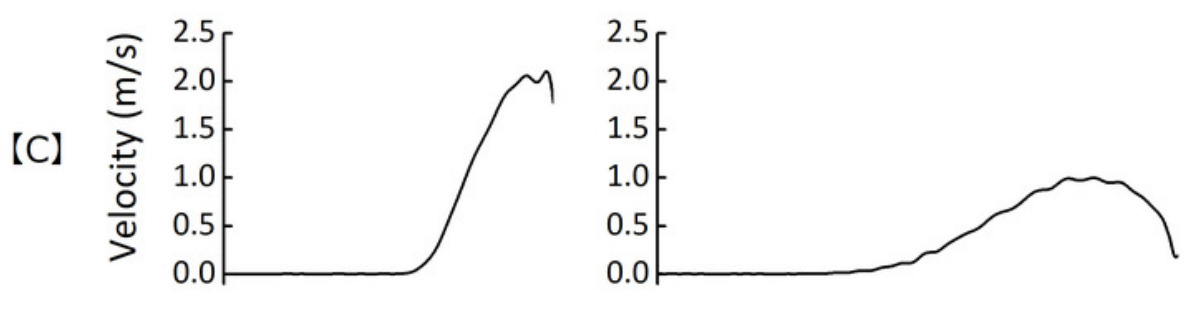
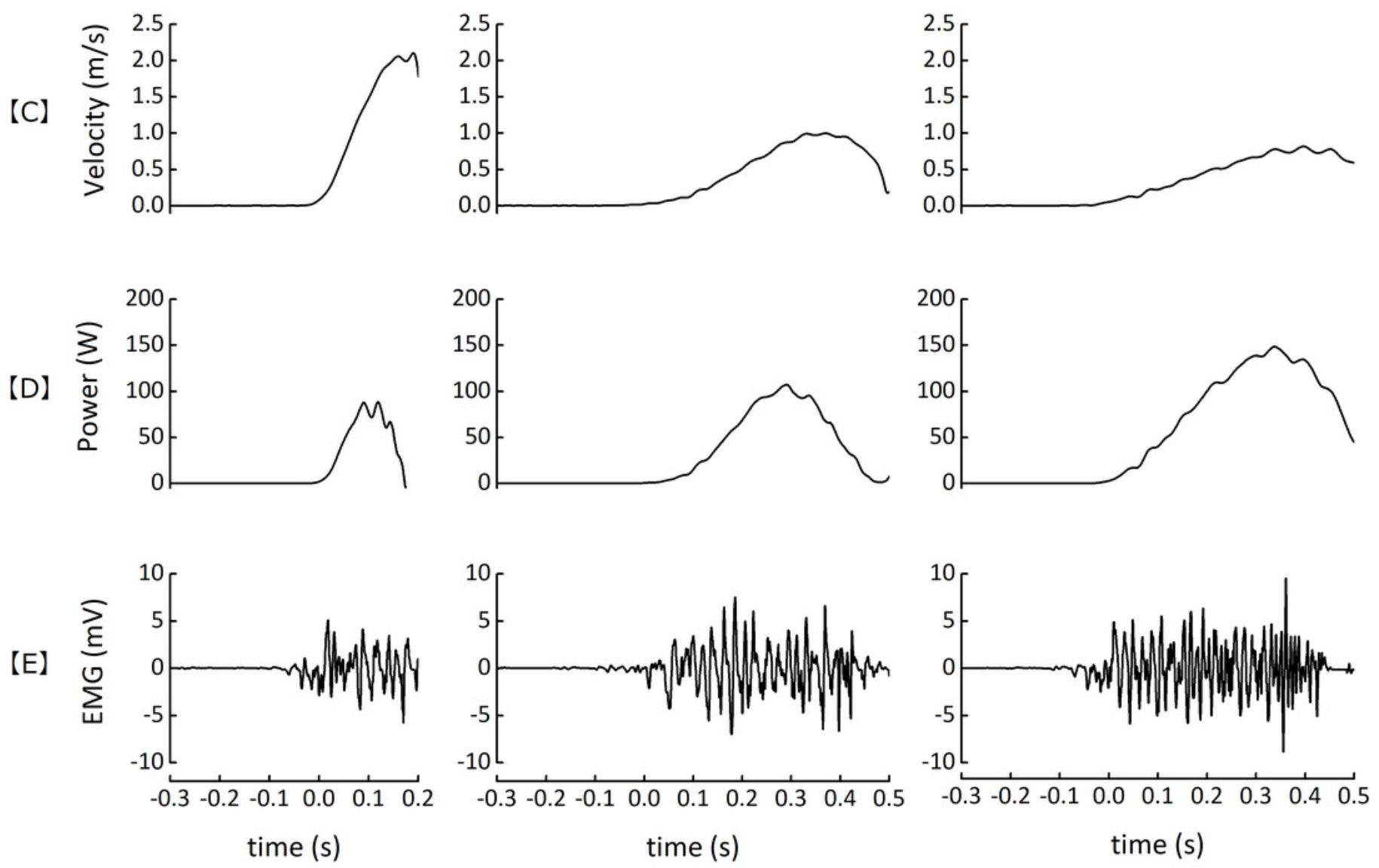
Figure 3

Force-velocity relationship and parameters.

The average values (A) and individual values (B). Force-velocity relationship and parameters derived from each of the two relationships of gymnasts (the closed circle) and judo athletes (the open circle). 


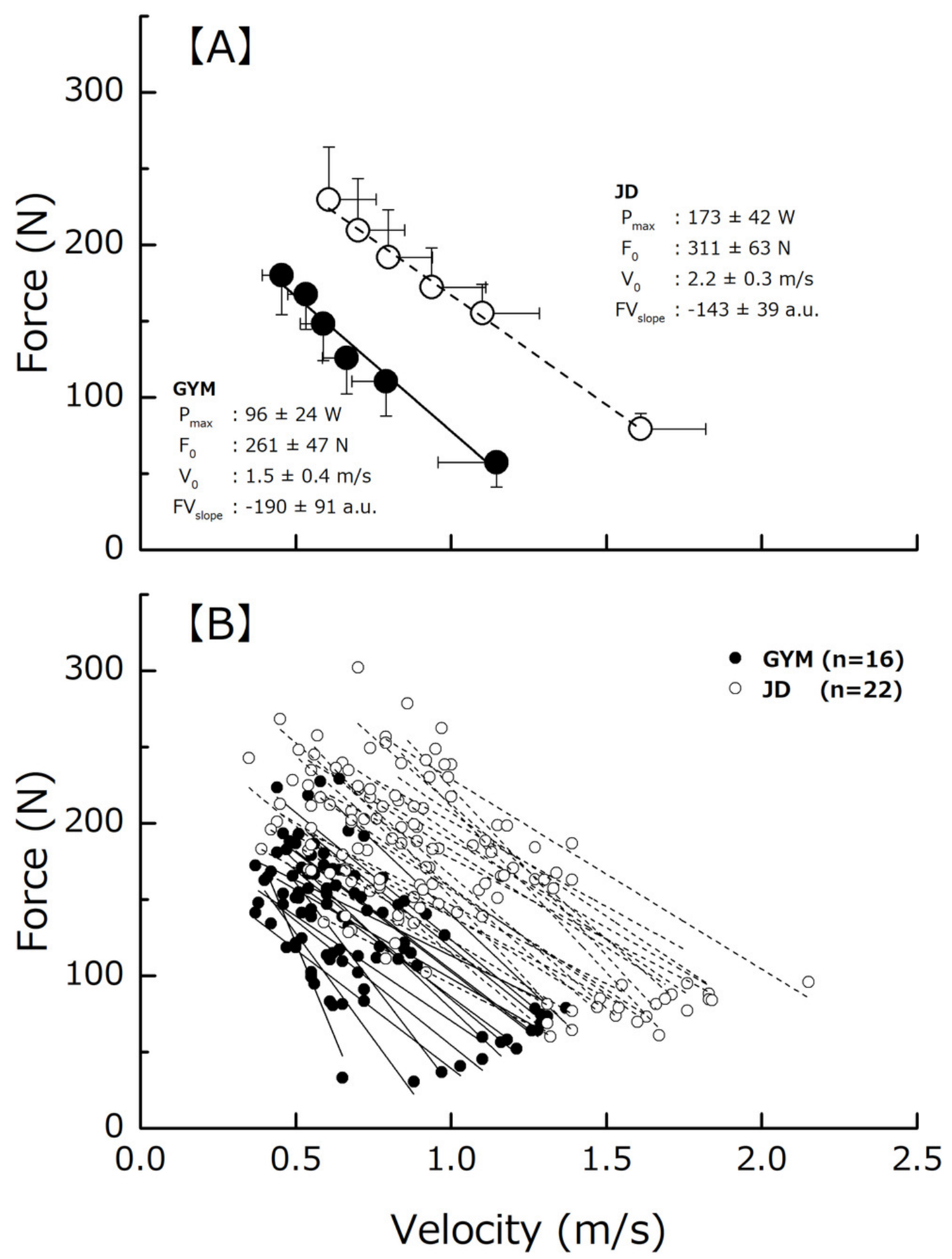

